\title{
Cost-Effectiveness of Somatostatin Analogues for the Treatment of Acromegaly in Colombia
}

\author{
Rafael Alfonso-Cristancho ${ }^{1,2}$, Santiago Herran Diazgranados ${ }^{2,3}$, Kariluz Maestre Martinez ${ }^{4}$, \\ Oscar David Diaz-Sotelo ${ }^{2}$ \\ ${ }^{1}$ Pharmaceutical Outcomes Research and Policy Program, University of Washington, Seattle, USA \\ ${ }^{2}$ RANDOM Foundation, Bogotá, Colombia \\ ${ }^{3}$ Health Economics and Pharmacoeconomics Program, Pompeu Fabra University, Barcelona, Spain \\ ${ }^{4}$ Novartis de Colombia, Rafael Alfonso, SA \\ Email: ralfonso@random-web.org
}

Received April 25, 2012; revised May 27, 2012; accepted June 28, 2012

\begin{abstract}
Background: Somatostatin analogues have shown to be effective in controlling the levels of growth hormone and are recommended for the treatment of acromegaly. These treatments have high costs of acquisition and their use might be restricted in limited resource settings. Objective: To evaluate the cost-effectiveness of somatostatin analogues for acromegaly in Colombia. Setting/Subjects/Intervention: A decision model was developed using a hypothetical cohort of patients with Acromegaly. Patients were treated according to the clinical practice of the country. Response to treatment and transition probabilities were derived from published literature. Costs and resource utilization were extracted from public and private sources in Colombia. Main Outcome Measure(s): Cost-effectiveness ratio, measured in Colombian pesos in a 2 year time-horizon. Results: The total medical treatment costs for the octreotide group were (Colombian Pesos) COP\$53,807,616, compared to the total costs for the lanreotide group of COP\$83,126,567. In the octreotide arm $65.30 \%$ of the patients and in the lanreotide arm $59.50 \%$ of the patients were successfully controlled. The number of deaths was 295 (13.1\%) and 302 (13.4\%) for octreotide and lanreotide, respectively. Because the costs are lower and the effectiveness is higher for octreotide in comparison with lanreotide, octreotide is more cost-effective than lanreotide. Probabilistic sensitivity analyses were consistent showing octreotide as the most cost-effective option. Conclusions: Costs and effects of octreotide compare favorably to those of lanreotide in the treatment of acromegaly in Colombia. Sensitivity analysis showed that despite the uncertainty in cost-effectiveness ratio this result is robust.
\end{abstract}

Keywords: Acromegaly; Somatostatin Analogues; Octeotride; Lanreotide; Cost-Effectiveness

\section{Introduction}

Acromegaly is a chronic condition characterized by excessive secretion of growth hormone (GH), which is mainly caused by pituitary tumors $[1,2]$. The incidence of this condition is three to four per million people and the prevalence is between 40 to 60 cases per million people [3]. The growth of these tumors is slow and delays the diagnosis of the disease by about 10 to 12 years until it is clinically recognized, more commonly in adults during their fourth or fifth decade of life [2-4]. The clinical features of acromegaly can be present in multiple forms, such as excessive growth in connective tissue, bones, and visceral organs, among others, accompanied by metabolic disorders, cardiovascular disease, sleep apnea, and colon neoplasms [5]. All these conditions and many others not described here but also present in acromegaly are associated with an increased mortality. According to a recent meta-analysis, all-cause mortality is almost dou- bled in subjects with acromegaly compared to the general population [6].

Current treatment algorithms and guidelines aim to control the levels of GH to $<2.5 \mu \mathrm{g} / \mathrm{l}$ and to lower the levels of the serum insulin-like growth factor-I (IGF-I) to the reference range according to the subject's age and gender. Achieving these goals leads to symptom resolution and less complications and mortality rates comparable to those of the general population [4,7]. To accomplish this goal multiple interventions could be required. The first step in small resectable tumors or large symptomatic tumors is a selective transsphenoidal surgery. The endocrine remission rate for this intervention is higher for microadenomas, around $70 \%$, compared to macroadenomas with less than $50 \%$. Major complication rates from this procedure are present in less than $10 \%$ of the patients and the mortality rate is less than 1\% [8]. Medical therapy is usually recommended following the 
surgery when biochemical remission is not achieved, nevertheless it can be used as primary therapy in patients with unresectable tumors, high surgical risk or who refuse surgery [9]. Two analogues of somatostatin, a hormone which inhibits GH, are available in Colombia: octreotide (Sandostatin $\mathrm{LAR}^{\circledR}$ ) and lanreotide (Somatuline $\left.\mathrm{ATG}^{\circledR}\right)$. In a recent metaanalysis, Murray et al., reported a greater biochemical efficacy for octreotide versus lanreotide for controlling the levels for GH (54\% vs. $48 \%$ ) and IGF-I (63\% vs. 42\%), nevertheless due to the methodological limitations of the studies included they did not find these differences to be significant in the control of biochemical markers [10]. These drugs have few side effects; most of them are gastric disturbances which resolve after the first months of therapy. In patients who do not achieve control levels with maximum doses of the somastostatin analogues, the addition of cabergoline, an oral dopamine agonist, is recommended. Adding cabergoline to any of the two somatostatin analogues, increases biochemical control by $21 \%$. Long-term exposure to cabergoline has been associated with increased risk of valvular heart disease [11].

In patients who do not respond to the previous treatments, conventional radiation therapy should be considered. Although the tumor growth and size is decreased with radiation, the biochemical control and symptoms improvement are slow, and may take 5 to 10 years to achieve reasonable control levels [12]. A previous step recommended by the guidelines, involves a $\mathrm{GH}$ receptor antagonist called pegvisomant but it is not available in Colombia.

Despite the guidelines and the evidence of the benefits of the treatment with somatostatin analogues for acromegaly, these treatments are not included in the mandatory health plan in Colombia [7], and their acquisition costs are high. Our aim is determine the cost-effectiveness of lanreotide and octreotide in the treatment of acromegaly in Colombia to inform decision makers and improve access to these treatments, optimizing health resources allocation.

\section{Methods}

\subsection{Model}

Following the current panel recommendations for Latin America [7], we developed a payer-based decision analysis model for the treatment of acromegaly in post-surgical patients with somatostatin analogues. After surgical resection of the tumor, a hypothetical cohort of patients is assigned to start treatment with octreotide LAR (20 - $30 \mathrm{mg}$ ) or lanreotide ATG (90 - $120 \mathrm{mg}$ ). If the serum GH level was controlled with the initial therapy, they continue their treatment with the same dose and medical follow-ups as scheduled. Those patients, who after six months fail to control GH serum levels to $<2.5$ $\mu \mathrm{g} / \mathrm{l}$, will have a dose increase and are followed six months later. If despite the dose increase, the patient is unable to achieve target $\mathrm{GH}$ serum levels, cabergoline $2.5 \mathrm{mg}$ is added to initial treatment with somatostatin analog. Finally, radiation therapy is available for patients who could not be controlled with combined drug therapy after another 6 months of therapy (Figure 1).

The minimum time horizon of the model was 2 years, assuming medical visits and dose adjustments every 6 months until GH level control was achieved or the patient is sent to radiation therapy, nevertheless the time horizon of the base case was 2 years.

\subsection{Clinical Outcomes}

The probability of achieving serum GH levels $<2.5$ with somatostatin analogues was extracted from the metaanalysis by Murray et al. [13], in which they reported that $65.3 \%$ of the patients treated with octreotide $59.5 \%$ of the patients treated with lanreotide were successfully controlled. The effect of the addition of cabergoline, as combined therapy, was considered to be the same in both somastostatin analogues, adding $21 \%$ of patients with normal levels of GH [14].

The annual mortality rate was calculated from the life tables of the Departamento Nacional de Estadistica (DANE) [15] and adjusted for the distribution of age and sex of the simulated cohort. For the group of patients who did not achieve control, we assumed a doubling of the mortality rate compared to the general population during the time they were uncontrolled until the end of follow-up. For patients who died during follow-up, an adjustment of resource use and costs was applied assuming only half the total annual cost during the last year of life during follow-up.

\section{Results}

Using the estimated prevalence of acromegaly in Colombia (55 cases per one million people), a hypothetical coHort of 2503 subjects with acromegaly were simulated. The average age of the cohort was 50 years and distribution by sex was equivalent. Of these we assumed that $90 \%$ of the subjects would have surgery and require treatment with a somatostatin analogue.

The total annual costs of management of acromegaly in Colombia, including physician visits, laboratory tests, and imaging were approximately COP\$2,264,010 per patient per year. The total annual costs of treatment per patient were lower for octreotide compared to lanreotide, and the total cumulative cost per patient after 2 years of follow up were also lower for octreotide (Table 1).

Since the total direct medical costs are lower and the 


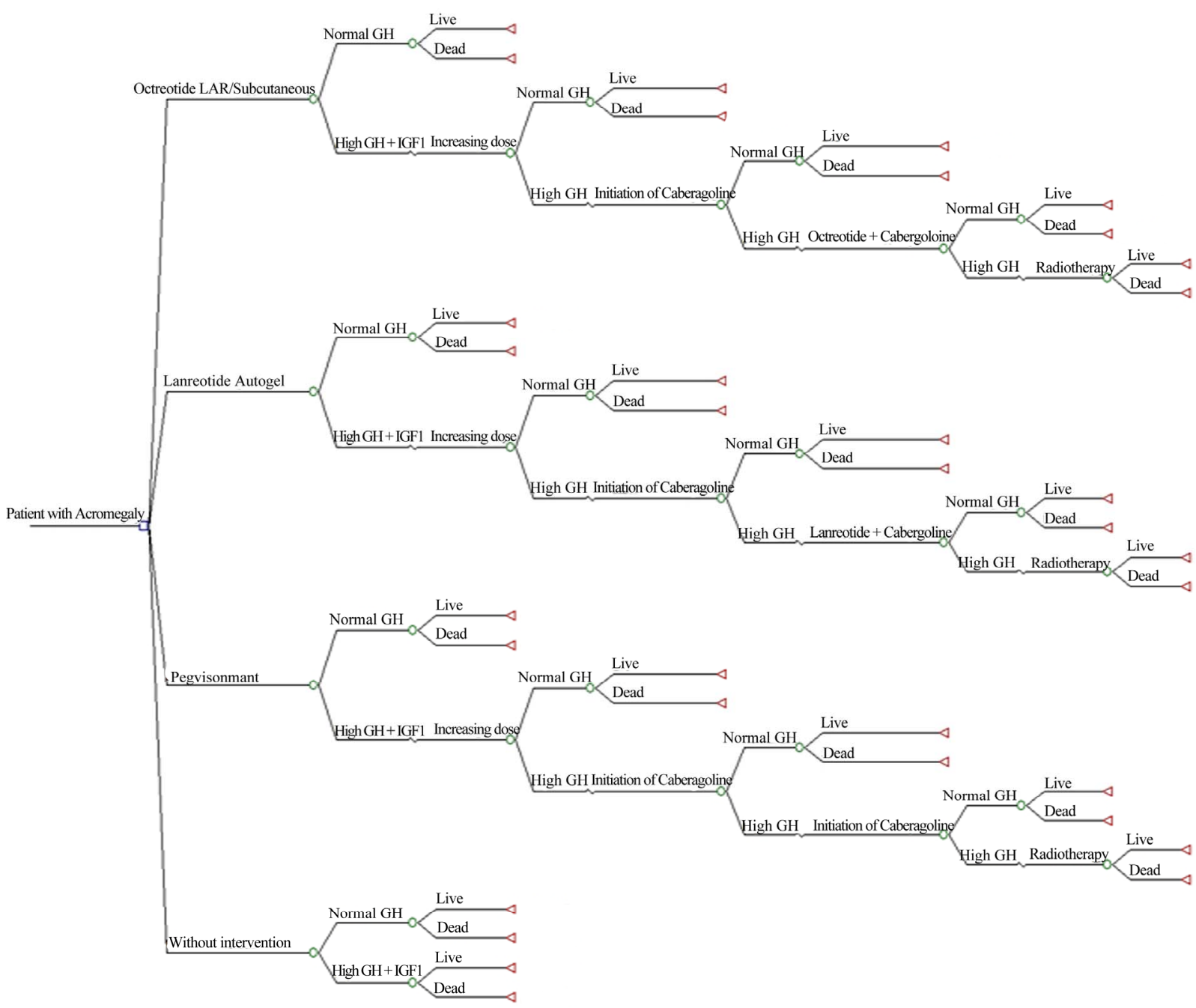

Figure 1. Decision tree for patients with Acromegaly.

Table 1. Results of costs analysis for Acromegaly managing in Colombia.

\begin{tabular}{ccccc}
\hline Therapy & Annual costs per patient & $\begin{array}{c}\text { Total cost cumulative per } \\
\text { patient }\end{array}$ & $\begin{array}{c}\text { Total cumulative mortality } \\
\text { Comulative number of } \\
\text { deaths }\end{array}$ & $\begin{array}{c}\text { Cum } \\
\text { Octeotride }\end{array}$ \\
Lanreotide & $\$ 53,807,616$ & $\$ 98,269,601$ & $13.12 \%$ & 295 \\
\hline
\end{tabular}

effectiveness in the proportion of patients achieving biochemical control is higher for octreotide in comparison with lanreotide, octreotide is dominant. According to the model results, when using octreotide, estimated savings for Colombian Health Care system could raise up to COP\$9,545,519 per patient, per year.

Univariate sensitivity analyses were consistent showing octreotide as the most cost-effective option in all the scenarios explored. In the sensitivity analysis, the change in the efficacy of the treatment alternatives causes the highest changes in the calculated ICER compared to the reference case, nevertheless octreotide remains dominant at all instances.

\section{Discussion}

Our results show that differences in the effectiveness and costs associated with the treatment of acromegaly with somatostatin analogues in Colombia favor the treatment with octreotide compared to lanreotide. Previous studies have reported similar results in other countries [16-18] Valentim et al. conducted a cost-effectiveness and impact budget analysis on the Brazilian health budget in 
patients with uncontrolled acromegaly after surgery [16]. The authors concluded that octeotride was dominant compared with lanreotide and avoided 12 cases of GH serum levels elevation, allowing additional savings of \$8089 per patient per year for the health system in Brazil. The economic analysis performed in the UK and France are more difficult to put into context since they were performed before many of the new evidence that support our model was published and at that time their approach was considering somatostatin analogues as an alternative to dopamine antagonists for the treatment of acromegaly.

The impact of the effectiveness of treatment for both molecules was the parameter that showed the greater variability in the sensitivity analysis, therefore a controlled clinical trial head to head between the two molecules with a comparable population is needed to reduce uncertainty in that parameter, and likewise, additional information with data from the clinical practice of real life in Colombia that would allow us to establish even more precise values for the frequency of dose and adherence to treatment would be very useful in our models because we had to make assumptions based on having no data available.

Currently, a project for the development of integrated clinical guidelines from the Ministry of Social Protection in Colombia includes economic evaluations and budget impact analysis to inform decision makers about the potential impact of their recommendations. Guidelines ensure for intra-pathology assessments for the economic and budget impact analysis. In the case of acromegaly, is important to consider these results to inform a budget impact model to assess the affordability of these treatments for Colombian Health Care System.

\section{Conclusions}

In Colombia, the treatment of acromegaly with octreotide LAR has lower costs and higher number of patients that achieve biochemical control compared to lanreotide ATG. Octreotide is still dominant under different scenarios in the sensitivity analysis showing robustness in the results of the model despite the uncertainty around some estimates.

Adequate treatment of Acromegaly patients can reduce morbidity and mortality associated costs, especially when evidence based clinical guidelines and cost-effective alternatives are used.

\section{REFERENCES}

[1] S. Melmed, "Acromegaly Pathogenesis and Treatment," 2009. http://www.ncbi.nlm.nih.gov/pubmed/19884662

[2] S. Melmed, "Medical Progress: Acromegaly,” 2006. http://www.ncbi.nlm.nih.gov/pubmed/17167139

[3] B. A. Bengtsson, S. Eden, I. Ernest, A. Odén and B.
Sjögren, "Epidemiology and Long-Term Survival in Acromegaly: A Study of 166 Cases Diagnosed between 1955 and 1984,” 1988.

http://www.ncbi.nlm.nih.gov/pubmed/3369313

[4] S. Melmed, A. Colao, A. Barkan, M. Molitch, A. B. Grossman and D. Kleinberg, "Guidelines for Acromegaly Management: An Update,” 2009.

http://www.ncbi.nlm.nih.gov/pubmed/19208732

[5] A. Colao, D. Ferone, P. Marzullo and G. Lombardi, "Systemic Complications of Acromegaly: Epidemiology, Pathogenesis, and Management,” 2004.

http://www.ncbi.nlm.nih.gov/pubmed/14769829

[6] O. Dekkers, N. Biermasz, A. Pereira, J. Romijn and J. Vandenbroucke, "Mortality in Acromegaly: A metaanalysis,” 2008.

http://www.ncbi.nlm.nih.gov/pubmed/17971431

[7] A. Barkan, M. D. Bronstein, O. D. Bruno, A. Cob, A. L. Espinosa de los Monteros and M. R. Gadelha, "Management of Acromegaly in Latin America: Expert Panel Recommendations,” 2010.

http://www.ncbi.nlm.nih.gov/pubmed?term=Management \%20of\%20acromegaly\%20in\%20latin\%20america\%3A\% 20Expert\%20panel\%20recommendations

[8] E. R. Laws, "Surgery for Acromegaly: Evolution of the Techniques and Outcomes," 2008. http://www.ncbi.nlm.nih.gov/pubmed/18228147

[9] R. Cozzi, M. Montini, R. Attanasio, M. Albizzi, G. Lasio and S. Lodrini, "Primary Treatment of Acromegaly with Octreotide LAR: A Long-Term (up to Nine Years) Prospective Study of Its Efficacy in the Control of Disease Activity and Tumor Shrinkage,” 2006.

http://www.ncbi.nlm.nih.gov/pubmed/16449332

[10] R. D. Murray and S. Melmed, "A Critical Analysis of Clinically Available Somatostatin Analog Formulations for Therapy of Acromegaly,” 2008.

http://www.ncbi.nlm.nih.gov/pubmed/18477663 doi:10.1210/jc.2008-0027

[11] U. P. Freda, C. M. Reyes, A. T. Nuruzzaman, R. E. Sundeen, A. G. Khandji and K. D. Post, "Cabergoline Therapy of Growth Hormone \& Growth Hormone/Prolactin Secreting Pituitary Tumors,” 2004.

http://www.ncbi.nlm.nih.gov/pubmed/15638294

[12] P. J. Jenkins, P. Bates, M. N. Carson, P. M. Stewart and J. A. Wass, "Conventional Pituitary Irradiation Is Effective in Lowering Serum Growth Hormone and Insulin-Like Growth Factor-I in Patients with Acromegaly,” 2006. http://www.ncbi.nlm.nih.gov/pubmed/16403824

[13] R. D. Murray and S. Melmed, “A Critical Analysis of Clinically Available Somatostatin Analog Formulations for Therapy of Acromegaly,” 2008. http://www.ncbi.nlm.nih.gov/pubmed/18477663 doi:10.1210/jc.2008-0027

[14] R. Cozzi, R. Attanasio, S. Lodrini and G. Lasio, “Cabergoline Addition to Depot Somatostatin Analogues in Resistant Acromegalic Patients: Efficacy and Lack of Predictive Value of Prolactin Status,” 2004. http://www.ncbi.nlm.nih.gov/pubmed/15272916

[15] Tabla Anual de Mortalidad, "DANE—Departamento Dministrative Nacional de Estadistica,” 2010. 
http://www.dane.gov.co/daneweb_V09/

[16] J. Valentim, V. Passos, F. Mataveli and A. Calabró, "Cost-Effectiveness Analysis of Somatostatin Analogues in the Treatment of Acromegaly in Brazil," 2008. http://www.ncbi.nlm.nih.gov/pubmed/19197453

[17] P. Chanson, "Predicting the Effects of Long-Term Medical Treatment in Acromegaly at What Cost? For What Benefits?” 1997. http://www.ncbi.nlm.nih.gov/pubmed/9150692

[18] D. Moore, C. Meads, L. Roberts, F. Song and E. Date, "The Effectiveness and Cost-Effectiveness of Somatostatin Analogues in the Treatment of Acromegaly," 2002.

http://www.crd.york.ac.uk/CRDWeb/ShowRecord.asp?ID $=32002000871$ 